\title{
Maximizing utilization of research
}

Frontiers in Reproductive Health

Follow this and additional works at: https://knowledgecommons.popcouncil.org/departments_sbsr-rh

Part of the Demography, Population, and Ecology Commons, Family, Life Course, and Society Commons, Health Services Research Commons, International Public Health Commons, and the Medicine and Health Commons How does access to this work benefit you? Let us know!

\section{Recommended Citation}

"Maximizing utilization of research," FRONTIERS Legacy Themes. Washington, DC: Population Council, 2009. 


\section{Population Council FRONTIERS IN REPRODUCTIVE HEALTH}

\section{Legacy Series.}

\section{MAXIMIZING}

\section{UTILIZATION OF RESEARCH}

This paper is part of a series of eight Legacy Papers synthesizing major lessons learned through research conducted under the Frontiers in Reproductive Health Program (FRONTIERS).

The full set of Legacy Papers includes:

-- Capacity Building

-- Family Planning

-- Female Genital Mutilation/Cutting

-- Gender

-- Integration of Services

-- Sustainability of Services

-- Utilization of Research Findings

-- Youth Reproductive Health

The complete reports referenced in these papers are available online:

www.popcouncil.org/frontiers

Moreover, an OR project may be expected to achieve several types of utilization, depending on project nature, generalizability of findings to other programs and settings, and availability of resources for more than one type of utilization. For example, an intervention proven successful in a district may first become institutionalized within that district's health program and then scaled up to other districts in the same province and to other provinces. If appropriate, it may also be replicated in another country.
Operations research (OR) can only be judged only as successful if results are utilized for making decisions to strengthen RH/FP policies and service delivery. How can this best be achieved? How can both process and impact of OR be measured? Drawing from 10 years of FRONTIERS OR experience, a number of key principles for promoting research utilization, illustrated with documented examples, are presented here.

One important first step is clarifying terms used, almost interchangeably, in research utilization, as it incorporates a range of ways in which research can be used for making decisions to strengthen RH/FP policies and programs. (Concepts underlying terms used are defined in Box 1.) Achieving, and measuring, utilization of research depends in part on what type(s) of utilization is envisioned, and so it is critical the specific type needs to be considered and specified before research begins.

\section{Box 1. Clarifying Research Utilization Language}

Research utilization: making decisions concerning policy, advocacy and resource allocation, planning and management, and program systems development and strengthening, using information generated from research.

Institutionalization: incorporation of a practice or intervention proven to be effective (sometimes termed a 'best practice') within the routine activities of a facility, program or organization.

Replication: introduction of a proven intervention or practice into another setting; this may be another program or another country.

Scale-up: extension of an intervention or proven practice beyond the original project site. 
Most of the principles described below are relevant whether research concerns introduction of a new or improved RH/FP technology to a country or program (such as emergency contraception), or of a new / revised service delivery guideline or tool (such as the Balanced Counseling Strategy or Systematic Screening or an educational or training curriculum), or the reorganization of service delivery systems (such as integrating FP with HIV services, or using community-level workers to provide services usually offered in clinics).

\section{Communicating Results to Influence Decisions}

Underlying all research utilization is communication of information generated by those who produce it to those who can use it for making decisions. FRONTIERS experience suggests a number of strategies can enhance likelihood this communication can be effective.

Translate and synthesize. Research, like service delivery, has its own vocabulary that can be difficult for non-researchers to understand. Moreover, the types of action decisions that could be made based on findings should be explicitly stated as recommendations; simply presenting research findings requires the decision-maker to interpret them, which may result in wrong decisions. Bringing together a range of evidence from several research studies can greatly strengthen findings. Documents such as the FRONTIERS series of Program Briefs (Box 2) are examples of how research findings can be translated and synthesized to produce programmatically useful information.

\section{Communicate results through multiple} channels to reach the same audience many times and many audiences at least once. Hearing the same message many times and from different sources increases likelihood it will be used. Communicating the same findings in Final Reports, OR Summaries (www.popcouncil.org/frontiers/pubs_types/ orsummaries/ors.html), Program Briefs, national workshops, international conferences, listserv announcements, interpersonal discussions and other forms of media has increased visibility of key findings from FRONTIERS projects.

\section{BoX 2. FRONTIERS Program Briefs}

FRONTIERS produced 13 Program Briefs, 4- to 20- page documents that synthesized findings from OR on major reproductive health issues, online at:

www.popcouncil.org/frontiers/ pubs types/prbriefs.html

1. Meeting women's needs after abortion

2. Using men as community-based distributors of condoms

3. Enhancing quality for clients: The balanced counseling strategy

4. Postabortion family planning benefits clients and providers

5. Building capacity to utilized operations research

6. Systematic screening: A strategy for determining and meeting clients' reproductive health needs

7. Make better use of provider time in public health clinics

8. How much will it cost to scale up a reproductive health pilot project?

9. Increasing women's use of the IUD for family planning

10. Meeting the family planning needs of postpartum women

11. Adapting focused antenatal care: Lessons from three African countries

12. Financial capacity building for NGO sustainability

13. Multisectoral youth interventions: The scale-up process in Kenya and Senegal 
Share results with specialist 'mediator' organizations. Recognizing the need for researchbased evidence and difficulties faced by most researchers in effectively communicating programmatic messages from findings, a number of specialist organizations and projects now exist that can assist in communicating findings (Askew, Matthews, and Partridge 2006). Examples include the Population Reference Bureau, the Johns Hopkins Center for Communications Programs, and the "C-Change" project, among others. A particularly useful mediator for RH/FP programming is the "Implementing Best Practices" initiative, coordinated by WHO (Box 3).

\section{Box 3. The IBP Initiative}

Initiated by WHO and USAID and with supported by numerous international agencies, the IBP Initiative seeks to improve access to evidence-based practices in reproductive health. The coalition supports country and regional conferences focused various issues, from identifying best practices on a specific topic to scaling up proven practices throughout the health care system. The IBP initiative has produced guidelines on the scale-up process, and also operates a "Knowledge Gateway" to circulation of documents on best practices and discussion of field experiences. The Knowledge Gateway offers community discussions on a range of topics, a community library, announcements, and a calendar of events. www.ibpinitiative.org

Become a decision-maker. One extremely effective way to communicate findings is for researchers themselves to engage in decision-making processes. Researchers can play extremely useful roles when serving on committees, steering groups, technical advisory groups, program design teams, etc. by bringing not only information from their own research but also a thorough understanding of literature around the topic.

Managers also have a responsibility to seek out research-based information. The onus on communicating research results is usually placed on those producing information. However, a decision-maker has to want to use such information, must know how to find it, and should actively search for it. FRONTIERS addressed this issue in a number of ways, including developing a short course on OR specifically tailored for managers (Box 4), engaging national decision-makers in designing and implementing OR projects, supporting participation at research conferences, and soliciting their perspectives on research priorities.

\section{Box 4. OR for Managers}

This three-day course is one of several introductory courses on OR developed by FRONTIERS. The OR for Managers course is designed to educate managers of reproductive health and service provision programs on operations research and how to apply research findings to improve programs.

www.popcouncil.org/frontiers/OR Course/index.htm 


\section{Strategies for Increasing Research Utilization}

While it is both impossible and inappropriate to propose a standardized model for developing and implementing OR to maximize utilization, experience from almost 200 projects over 10 years has provided FRONTIERS with many examples of what does and does not work. This experience has been combined with WHO's and other partners' experiences in "Turning Research into Practice" (Box 5). FRONTIERS also collaborated with several UKbased DFID-funded applied research groups, which provide another rich source of guidance on maximizing research utilization (Askew, Matthews, and Partridge 2006; Nath 2007). The MEASURE Evaluation Project's 'Data Demand and Information Use' initiative also offers a useful framework and several tools for ensuring research can inform program decision makers (Foreit et al. 2006). Some of the key strategies emerging from these reviews follow.

\section{Plan for utilization BEFORE starting research.} Managers and researchers must consider longterm prospects for research before obtaining funding and even before writing research proposals. Planning needs to address several questions: Who will use the data? What decisions can be influenced? Can they commit themselves to making and funding changes needed?

\section{Engage and work with data users and other stakeholders throughout research} process. Involve those who will use the data (managers, clinicians, policymakers) in research design to find out what type of data would be most persuasive and effective. Give regular progress reports (in person and writing) and encourage managers to make site visits. Work with them to interpret draft results before finalizing the study so they can understand and identify programmatic implications.

\section{Undertake operations research in a favorable}

context. Valid measures of feasibility and effectiveness can only be achieved when an intervention is fully implemented. Thus it is preferable to select project sites from among those that are stable, functioning, and ready to deliver intervention.

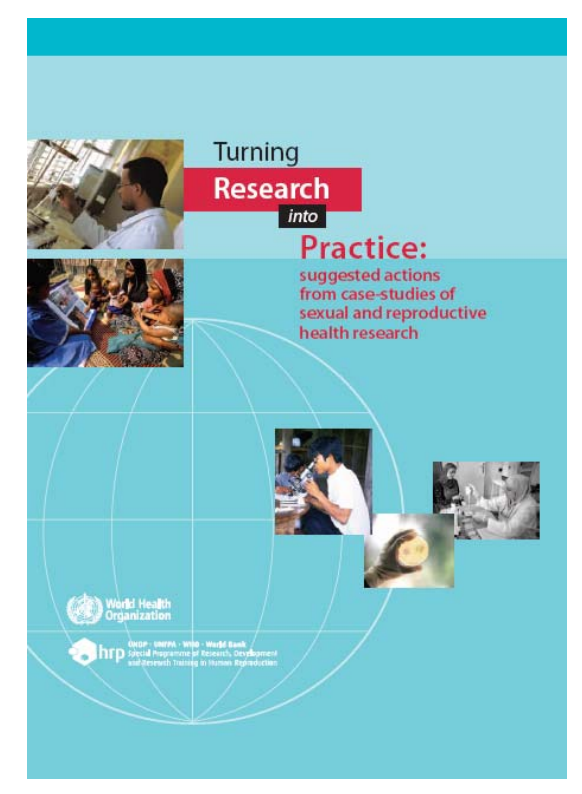

Box 5. TRIP Report: A framework for maximizing research utilization

This report is the product of two international meetings in which researchers, policymakers, donors, and program managers developed guidance on increase the use of research findings in program development and monitor the incorporation of evidence-based practices within reproductive health programs.

www.who.int/reproductivehealth/publications/trip/index.html 
Use the strongest research design and data collection methods possible. Fully experimental designs are not always possible, but strong quasi-experimental designs should be used wherever possible. Adhering to national and international ethical standards for research must be a priority, even if it compromises ideal study design. Be careful not to collect too much data; be guided by types of programmatic decisions you would like data to inform.

Analyze data quickly and prioritize results needed for decisions. Keep initial analysis simple, yet appropriate. Involve decision-makers in data analysis and interpretation so findings can be translated into actionable statements or policies. Prioritize communication of findings to decision-makers most likely to use findings and to communities participating in studies first.

Plan for, budget for, and include "utilization" phase within research process. OR strengthening service delivery should not end with results dissemination to decisionmakers; it is unreasonable to expect them to simply turn findings into action. Resources should be leveraged so those who undertook pilot project can provide technical assistance to help programs implement changes indicated by research findings.

\section{Only recommend and advocate for service delivery changes if results really do} demonstrate feasibility, effectiveness (and preferably cost-effectiveness), and potential for institutionalization and scale-up. Research data can be misunderstood or misused-for example, to support particular viewpoints. Great care is needed to avoid recommending interventions be adopted when data does not really demonstrate convincing effectiveness, as well as approaches to service delivery being changed unless alternatives really are more effective or less costly.

\section{Institutionalization of Proven Practices: Critical Step in Creating Conditions for Scaling Up Effective Interventions}

Technical assistance is not usually conceptualized as a "research" activity. However, after research has identified a promising practice, a follow-on phase of technical assistance is an effective strategy for enhancing institutionalization of a new practice prior to full scale-up. During this follow-on phase, individuals or organizations who undertook the pilot project provide support to help the RH/FP program enact changes necessary to institutionalize the practice, so it becomes routine. Within FRONTIERS, this adaptation phase was organized as and termed a "creating conditions for scale-up" project. These projects lasted from six to 18 months depending on the adaptations needed, and could include activities such as reorganizing staff responsibilities and skills training in new procedures, revising training curricula, changing supervisory procedures, restructuring recordkeeping and reporting forms, and so on. 
To ensure interventions proven effective during pilot projects can be institutionalized into program routine operating procedures, "creating conditions" projects support expansion of procedures throughout all facilities in one or more districts - and usually district(s) in which the intervention was piloted. In most countries of Africa and South Asia, the district is the lowest level of health services administration; consequently, these projects provide technical assistance to staff of a district health management team (or its equivalent) by helping comprehensively institutionalize new or improved practices-that is, within district planning, budgeting, implementation, and reporting systems. Experience has shown this phase of limited scale-up of proven practices demonstrates the intervention can be incorporated into and funded by existing health systems, institutionalized into routine procedures, implemented at scale, and funded by the health program.

This phase has proven critical for an RH/FP program to learn how to perform these functions before more widespread scale-up can be undertaken. Examples of "creating conditions" projects include: a male RH services model in Bangladesh (Mannan et al. 2008); a Quality Assurance approach in Gujarat State, India (Khan et al. 2008); a model for involving men in maternity care in New Delhi, India (Varkey, Mishra, and Khan 2008); and community midwifery services in Western Province, Kenya (Mwangi and Warren 2008).

\section{Scaling up best practices: Effectiveness, efficiency, and expansion}

There are many reasons why pilot projects showing how to effectively implement new and improved practices are not scaled up, including limited funding (availability for duration of research project only); lack of expectation by donor those implementing pilot project should scale up practices tested; and an assumption service delivery organizations are able to take research findings and scale them up in an RH/FP program. Introducing a "creating conditions" phase enables programs to move from providing services effectively to learning how to provide them efficiently, by adapting new practices to existing district-level systems. Scaling-up from district level to regional or provincial, and then national levels, requires another phase: learning how to expand responsibility to institutionalize service delivery protocols throughout the service delivery program.

FRONTIERS experience with nationwide scale-up of emergency contraception (EC) services in Bangladesh (Khan and Hossain Forthcoming) and of expanding an adolescent RH services in Kenya (Evelia et al. 2008; Joyce et al. 2008) provide two examples of ways in which programs have learned to expand implementation of interventions proven effective and efficient. Some general lessons emerging from these two experiences include:

$\checkmark$ Ensuring the government ministry or ministries lead the process, not just in title but by designating key managers to make all relevant decisions;

$\checkmark$ Convincing the government to commit funding to sustain new way of providing services, whether from internal commitments or negotiations with development partners; 
$\checkmark$ Engaging a wide range of national and international stakeholders to appear not to advocate for one organization's interests;

$\checkmark$ Supporting policy and systems reviews and revisions facilitating nationwide implementation of new services and procedures;

$\checkmark$ Creating a training cascade through training of trainers at several levels to minimize resources needed.

An important lesson, for both those conducting a pilot project and for those responsible for scale-up, is testing and implementing only interventions a national program can afford to scale up. There is no point in pilot-testing interventions not affordable at scale. When designing a pilot project, planners should attempt to estimate costs of scaling up before embarking on 'creating conditions' and 'expansion' phases. In so doing, waste associated with piloting unsustainable

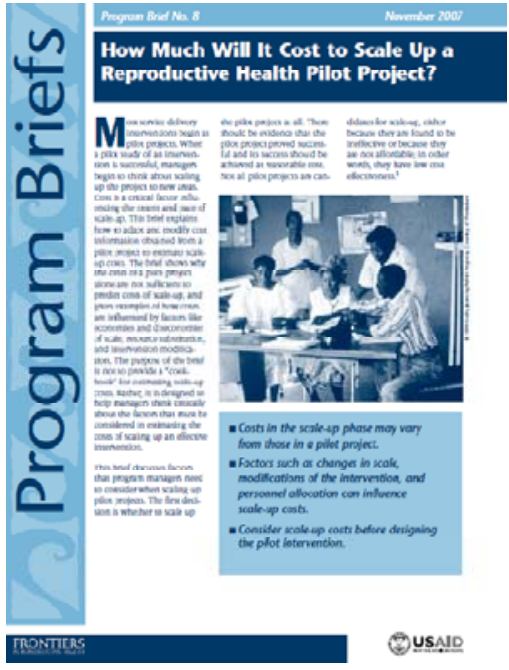

Guidance for adapting and modifying cost information obtained from a pilot project to estimate scale-up costs is provided in FRONTIERS Program Brief no. 8 (Janowitz, Bratt, Homan, and Foreit 2007). The brief shows why the costs of a pilot project alone are not sufficient to predict costs of scale-up, and gives examples of how costs are influenced by factors like economies and diseconomies of scale, resource substitution, and intervention modification. interventions can be avoided. Affordable interventions may produce less spectacular results during the pilot phase, but being able to scale up more modest, affordable interventions will make larger health impacts than small-scale projects yielding large health benefits but only in intensively-resourced pilot projects -so-called 'boutique' projects.

\section{Replicating Successful Interventions}

An intervention that is proven effective and efficient in one setting can be attractive to programs in other countries. An effective strategy to facilitate replication is to hold a workshop or conference, attended by policymakers and program managers from several countries in a region, at which practical guidance in introducing and scaling-up a proven best practice is provided by those responsible for successfully piloting the intervention. FRONTIERS used this strategy to replicate several of the interventions initially tested through OR projects.

In 2002 FRONTIERS joined a consortium of international organizations in Senegal to convene a regional conference that advocated for increasing access to and strengthening quality of postabortion care (PAC) services (Postabortion Care (PAC) Initiative for Francophone Africa Committee 2004) (Box 6). Two key presentations were the experiences of Senegal and Burkina Faso in developing and testing PAC interventions through OR projects (Askew 2006). 
These systematically documented pilot projects formed the basis of the model developed for introducing PAC services in other countries in the region. An analysis of the experiences of introducing PAC into four other countries (Guinea, Mali, Niger and Togo) and of scaling up the pilot experiences in Burkina Faso and Senegal by the Centre de Formation et de Recherche en Santé de la Reproduction (Center for Training in Reproductive Health Research or CEFOREP) describes the processes followed in the region (see box).

FRONTIERS used this strategy to replicate introduction of a gender accreditation tool developed and scaled-up in Bolivia (Palenque et al. 2004; Palenque, Riveros-Hamel, and Vernon 2007). In June 2007 a workshop in Costa Rica trained 31 participants in the strategy from Ministries of Health, Social Security Institutes, multilateral organizations and several NGOs in Bolivia, Peru, Ecuador, Dominican Republic, El Salvador, Honduras, Guatemala, and Costa Rica. Subsequently, technical assistance was provided to organizations in El Salvador, Honduras and Peru to support introduction of the tool (Riveros-Hamel, Martin, and Vernon 2008).

The same strategy has also been used recently in collaboration with WHO's Africa Regional Bureau for replicating a multisectoral adolescent $\mathrm{RH}$ model in several African countries (Burkina Faso, Ghana, Kenya, Mauritania, Mozambique, Namibia, Senegal, Tanzania, and Zimbabwe) (Diop and Diagne 2008), and for enhancing postpartum/postabortion family planning services in the Arab region (Egypt, Jordan, Sudan, and Yemen) (FRONTIERS 2008).

\section{Evaluating OR Utilization}

As with service delivery projects, evaluating operations research activities has many benefits:

$\checkmark$ Increasing recognition of value of evidence-based policy making and services programming;

$\checkmark$ Demonstrating to funder research is "making a difference" and is therefore a worthwhile investment;

$\checkmark$ Justifies allocation of resources and prioritizes future allocations;

$\checkmark$ Focuses researcher attention on utilization and application of research findings;

$\checkmark$ Helps improve design and implementation of research, thereby increasing likelihood of producing results than can be utilized. 
A number of ways have been tested to measure and document research utilization.

The case-study approach reviews utilization of a specific OR activity in great depth to understand whether findings were used, and if so, how. The "Getting Research into Policy and Practice" (GRIPP) initiative collected 18 case studies, documented researcher activities promoting utilization, and created a web portal for communicating experiences. GRIPP was a partnership between FRONTIERS, John Snow International (Europe), and two DFID-funded research programs, Opportunities and Choices and Safe Passages to Adulthood. A synthesis of the 18 case studies identified a number of factors facilitating utilization (Nath 2007). The case study approach requires documentation be integral to the research process from inception, a neutral facilitator document the process, adequate resources budgeted for documentation, and identification of appropriate stakeholders to elicit different perspectives.

A second approach reviews a broad portfolio of research initiatives undertaken in a particular setting to learn about underlying patterns facilitating or obstructing research utilization. Such an approach has been used in Mexico and Guatemala, Bangladesh, Indonesia, and Egypt. In Mexico, the focus was on describing the relationship between health researchers and policy makers and "to reconstruct the processes through which research was used to make decisions and policies" (Trostle 2006). In Guatemala, researchers reviewed 44 OR projects conducted by the Population Council between 1988 and 2001 (Brambila et al. 2007). Projects were on a wide range of topics, covered different target groups, and were conducted with diverse collaborating institutions and researchers. This approach has previously been used in Bangladesh (Hagga and Maru 1996), Egypt (Hegazi 1997) and Indonesia (Iskandar and Indrawati 1996).

A third approach generates quantitative measures of the extent to which lessons from an OR project have been used. Tulane University developed a methodology for assessing utilization of FRONTIERS projects that collected data on 14 process indicators (level of participation of key stakeholders, quality of research, problems in program implementation) and 11 impact indicators (Box 7), and six contextual factors (e.g. facilitating factors, barriers, assessment of costs) (Marin and Bertrand 2000; Marin, Gage, and Khan 2004). This methodology evaluated the extent of utilization of 64 FRONTIERS projects that had tested an intervention between 1998 and 2004 (RamaRao and Golon 2005). The Tulane methodology is easy to use, fairly low-cost and generates quantitative indicators, making it attractive to those funding research programs. For more textured process information, this methodology can be complemented by case studies and portfolio reviews.

\section{Box 7. Key Impact Outcomes Measured by Tulane Methodology:}

1. Were improvements made to program?

2. (If proven effective) were the improvements scaled up?

3. (If proven successful) were the improvements replicated?

4. Was policy (re-) formulated?

5. Was increased funding made available?

6. Was organizational capacity enhanced? 